\title{
Positron Annihilation in Magnetite Nanopowders Prepared by Co-Precipitation Method
}

\author{
K. Durak ${ }^{a}$, M. Wiertel ${ }^{a, *}, Z$. Surowiec ${ }^{a}$ And A. Miaskowski ${ }^{b}$ \\ ${ }^{a}$ Department of Nuclear Methods, Faculty of Mathematics, Physics and Computer Science, \\ M. Curie-Skłodowska University, pl. M. Curie-Skłodowskiej 1, 20-031 Lublin, Poland \\ ${ }^{b}$ Department of Applied Mathematics and Computer Science, University of Life Sciences in Lublin, Lublin, Poland
}

\begin{abstract}
Nanosized iron oxide powders are materials considered with regard to its application in medical therapy called hyperthermia. Magnetite nanopowders with crystallite size varying from 6.6 to $11.8 \mathrm{~nm}$ have been prepared by the co-precipitation method. In this study a change of a crystallite size is driven mainly by varying of initial $\mathrm{pH}$ of water ammonia solution in which a process of magnetite precipitation runs. Crystallographic structures and phase composition obtained samples and the size of magnetite nanoparticles were determined by X-ray diffraction method. Positron lifetime spectroscopy has been used to assess defectiveness of microstructure. Experimental positron annihilation spectra were successfully resolved into three lifetime components. It appears that from point of view of microstructure the defects concentrations in studied nanopowder samples are very high which causes a saturation of positron trapping.
\end{abstract}

DOI: 10.12693/APhysPolA.132.1593

PACS/topics: 81.07.Wx, 47.65.Cb, 78.70.Bj, 61.72.J-, 61.72.Mm, 68.37.Hk

\section{Introduction}

Magnetic nanoparticles (MNPs) of iron oxides are increasingly used in laboratory preparation and medical technologies such as hyperthermia, magnetic resonance imaging or magnetic drug delivery [1-4]. Popular in medical applications are magnetite $\left(\mathrm{Fe}_{3} \mathrm{O}_{4}\right)$ and maghemite $\left(\gamma-\mathrm{Fe}_{2} \mathrm{O}_{3}\right)$ nanoparticles. They enjoy great interest because of their low toxicity, biocompatibility, long time retention in blood and surface modifications. In addition, they can be metabolized by heme oxygenase- 1 to produce hemoglobin in the blood, which contributes to maintaining iron homeostasis in the cell [5]. The iron oxides nanoparticles can be obtained by various chemical methods e.g. vacuum spraying, surface redox exchange, hydrothermal process, sol-gel synthesis or liquid phase condensation [6]. Among various chemical methods for synthesis of different types of nanoparticles, co-precipitation route has several advantages over other methods, including good homogeneity, low cost, high purity of product, and not requiring organic solvents and heat treatment [7]. It consists in mixing the $\mathrm{Fe}^{2+}$ and $\mathrm{Fe}^{3+}$ iron from aqueous solutions of their salts (e.g. chlorides) in alkaline medium. An important aspect when obtaining nanoparticles is to control their size by selecting the synthesis parameters, among others: salt selection, determination of the $\mathrm{Fe}^{2+} / \mathrm{Fe}^{3+}$ ion ratio, reaction temperature, $\mathrm{pH}$ value and ionic strength of the solvent. Size of MNPs strongly affects their magnetic properties [8]. Nanocrystallites of magnetite that reach sizes of about $10 \mathrm{~nm}$ exhibit superparamagnetism, then they are con-

*corresponding author; e-mail: marek.wiertel@umcs.pl sidered as single domains having their magnetic moments which, under the influence of an external variable magnetic field, start to fluctuate. This particular property was used in the aforementioned hyperthermia [9]. The etymological meaning of "hyperthermia" is based on the heat generated at the site of the cancer. The temperature of the local tumor environment causes changes in the physiology of diseased cells, which ultimately leads to their apoptosis. Typically, the temperature increase is between $41^{\circ} \mathrm{C}$ and $46^{\circ} \mathrm{C}$, which contributes to intraand extracellular degradation processes such as protein denaturation or aggregation. The effectiveness of hyperthermia depends on the temperature produced at the target sites, exposure time and tumor specificity [10]. In case of single-domain nanoparticles, heat is generated mainly by two processes: the Neel relaxation and the Brown relaxation. The first is based on changes in orientation of the magnetic spins, and consequently magnetization within each nanoparticle, while in the Brown relaxation the MNPs can be rotated as a whole, which depends on both the hydrodynamic parameters of the particle and the surrounding medium [9].

This paper focuses on the characteristics of prepared magnetite nanoparticles by co-precipitation method. Xray diffraction (XRD) was performed to verify the crystallographic structure. The morphology of the obtained samples was observed on SEM micrographs. Moreover, for a detection and identification defects occurring in microstructure of samples the positron annihilation lifetime spectroscopy (PALS) was used. PALS is one of the methods suitable to estimate defect concentration.

\section{Experimental}

The synthesis of magnetite nanopowders were based on co-precipitation method in alkaline medium. The fol- 
lowing pure chemicals were used: $\mathrm{FeCl}_{2} \cdot 4 \mathrm{H}_{2} \mathrm{O}$ (Aldrich, $99 \%$ ), $\mathrm{FeCl}_{3} \cdot 6 \mathrm{H}_{2} \mathrm{O}$ (Aldrich, $98 \%$ ), $\mathrm{NH}_{4} \mathrm{OH} 25 \%$, distilled water. Firstly appropriate, stoichiometric amounts of ferric and ferrous chlorides were prepared in water solutions. Then, a solution of the ammonium base of the desired concentration was prepared. In the final step, a mixture of solutions of iron salts was added dropwise to the base solution under vigorous stirring (500 rpm). Obtained ferrofluid was washed with distilled water in three $30 \mathrm{ml}$ series and dried at room temperature (RT). There was four samples prepared with different concentration of ammonium base. Surface morphology of as-synthesized $\mathrm{Fe}_{3} \mathrm{O}_{4}$ nanoparticles was studied by means of scanning electron microscope Tescan Vega3 LMU at HV= $20 \mathrm{kV}$ working in secondary electron mode.

The XRD patterns were measured by means of a Philips X'Pert PW 3040/60 using diffractometer with $\mathrm{Cu} K_{\alpha}$ radiation at $\mathrm{RT}$. They were fitted using the FullProf program for the Rietveld refinement method.

In PALS measurements, the ${ }^{22} \mathrm{Na}$ source of $100 \mathrm{kBq}$ activity enclosed in Kapton envelope was used. A standard positron lifetime fast-slow spectrometer, equipped with two detectors with $\mathrm{BaF}_{2}$ scintillators was used. Time resolution of the spectrometer was about 250 ps (FWHM ${ }^{22} \mathrm{Na}$ ). All measurements are performed at room temperature and at vacuum $\left(\approx 5 \times 10^{-4} \mathrm{~Pa}\right)$. The total count number for each investigated sample amounts to at least $10^{7}$. Positron annihilation spectra were analysed using PALSfit program [11].

The thermal properties and usefulness of the synthesized nanopowders for application in hyperthermia were tested using the NanoTherics Magnetherm device [12].

\section{Results and discussion}

Figure 1 illustrates the SEM micrographs for the sample S2 at two different magnifications. The SEM image performed at magnifications of $500 \times$ shows a powder sample obtained directly after drying of aqueous solution of nanoparticles. The dried sample forms large (about $200 \mu \mathrm{m})$ agglomerates.

SEM image at a magnification of 330000 reveals that the samples consist of nanoparticles with a nearly spherical shape. They are approximately $8 \div 10 \mathrm{~nm}$ in size, indicating that almost homogeneous magnetite nanoparticles can be synthesized. However, the magnetite nanoparticles are relatively strongly agglomerated.

The crystal structures of obtained samples were verified by powder XRD. The $2 \theta$ values were taken from $20^{\circ}$ to $80^{\circ}$ using $\mathrm{Cu} K_{\alpha}$ radiation. Figure 2 shows the XRD patterns for four prepared samples of nanosized magnetite and the reference pattern of bulk magnetite. The shapes and arrangement of main reflexes for all samples is analogous and indicates the existence of the same phase. No additional peaks connected with polycrystalline magnetite were found in the resulting spectra. Visible differences appear in the width of the diffraction peaks due to the varied sizes of nanocrystallites. The strongly broad-
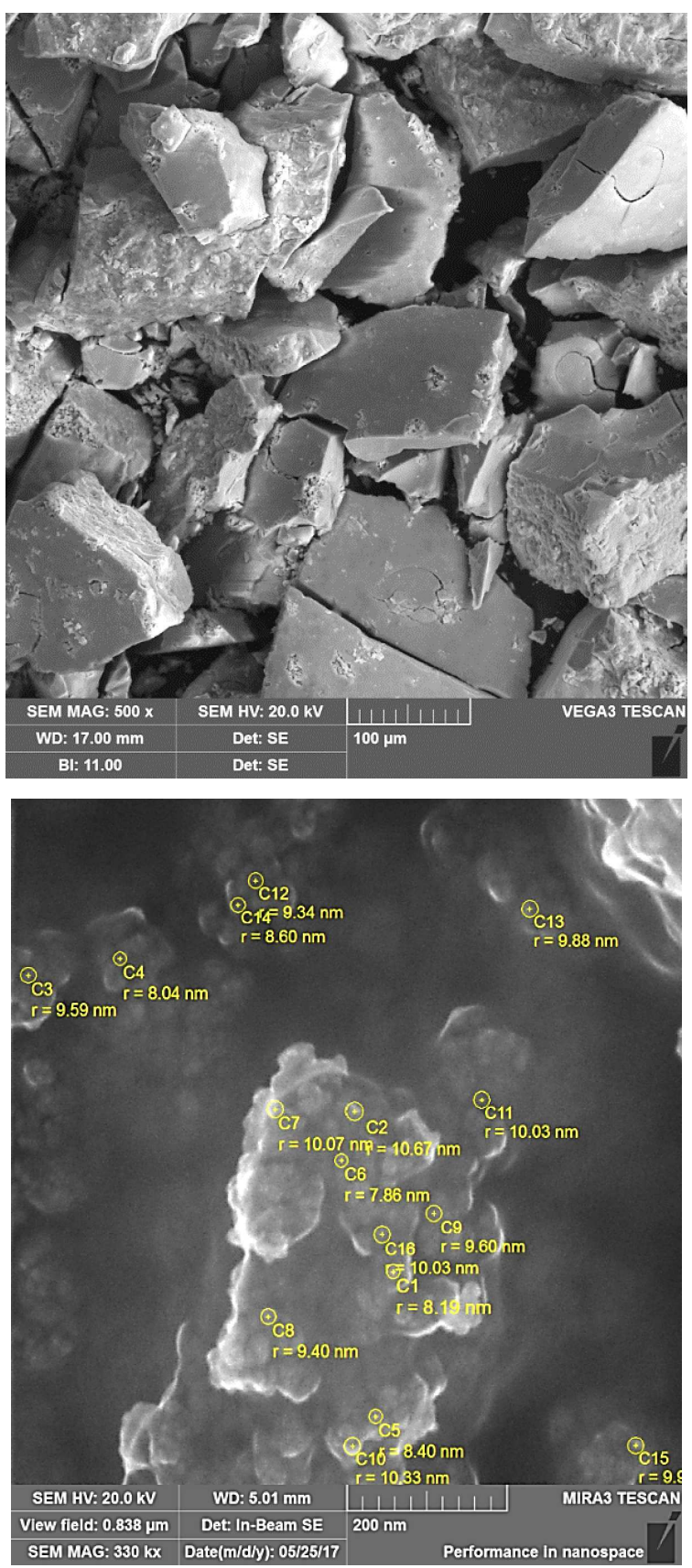

Fig. 1. SEM images at a magnification 500 and 330000 times, showing the agglomerates of nanoparticles (left) and nanoparticles shape and size distribution (right) for the magnetite powder synthesized by co-precipitation from $\left(\mathrm{FeCl}_{2} / \mathrm{FeCl}_{3}\right)$.

ened peaks in X-ray diffraction patterns are characteristic for nanoparticles.

The crystalline size and strain were calculated from the Williamson-Hall analysis.

As shown in Fig. 3 the size of nanoparticles strongly depends on the concentration of ammonium base. The crystallite size decreases linearly with increasing of ammonia concentration. 


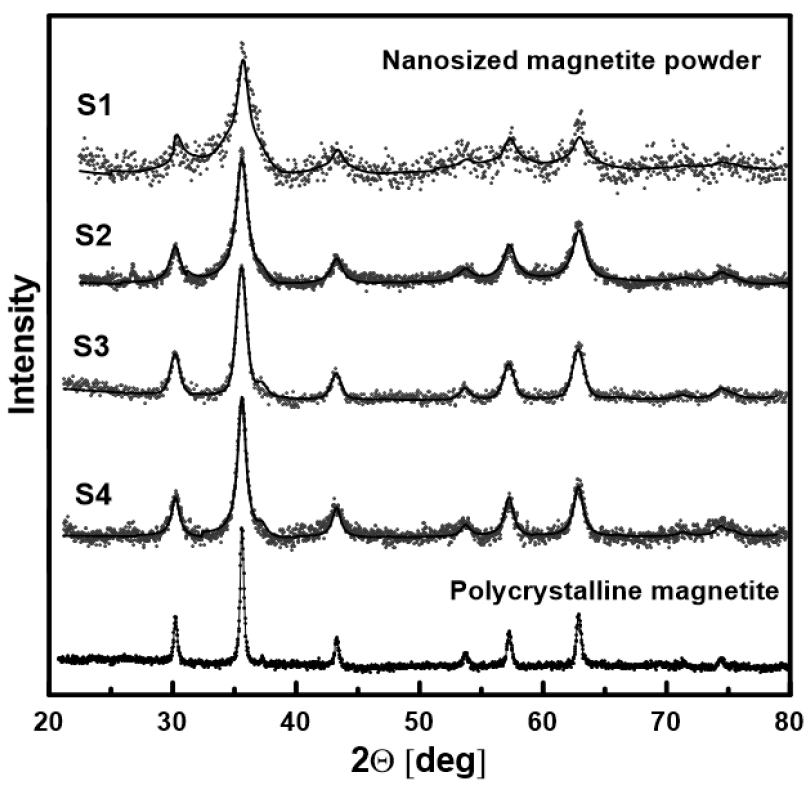

Fig. 2. The XRD patterns for nanosized magnetite powder and polycrystalline magnetite.

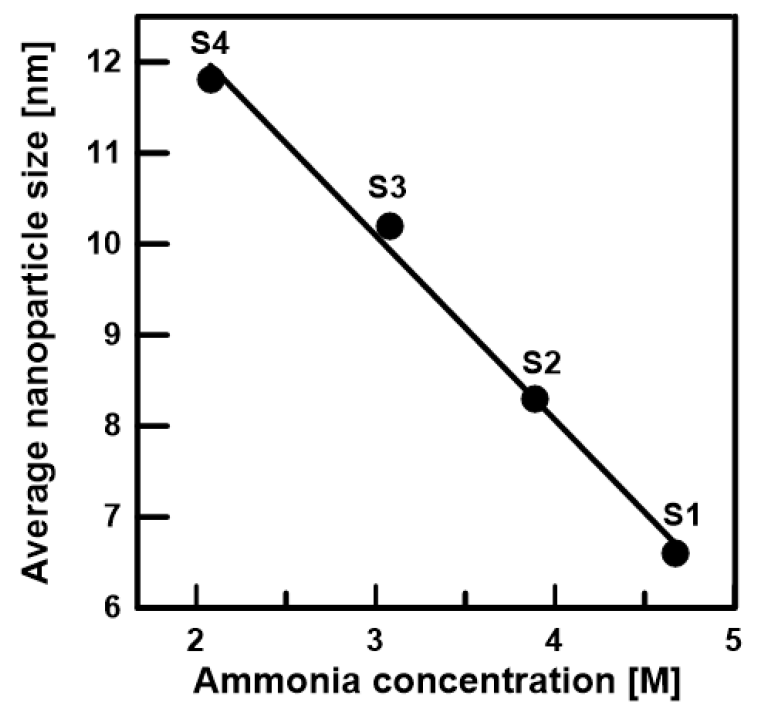

Fig. 3. The average magnetite nanoparticle sizes versus ammonia concentration.

This result shows that the size of magnetite nanoparticles can be well controlled, and stabilized, by adjusting the precipitation conditions.

Satisfactory accuracy $\left(\chi^{2}\right.$ of order $\left.1.1 \div 1.2\right)$ of numerical deconvolutions of PALS spectra into three exponential components was obtained. The third component of $\tau_{3} \approx 1.6 \mathrm{~ns}$ and $I_{3} \approx 0.2 \%$ origins from pick-off annihilation of ortho-Ps formed at sample grains surfaces and ${ }^{22} \mathrm{Na}$ source components [13]. In further considerations this component is neglected. In Fig. 4 mean lifetimes of two main components dependences on an average nanoparticle size were shown.

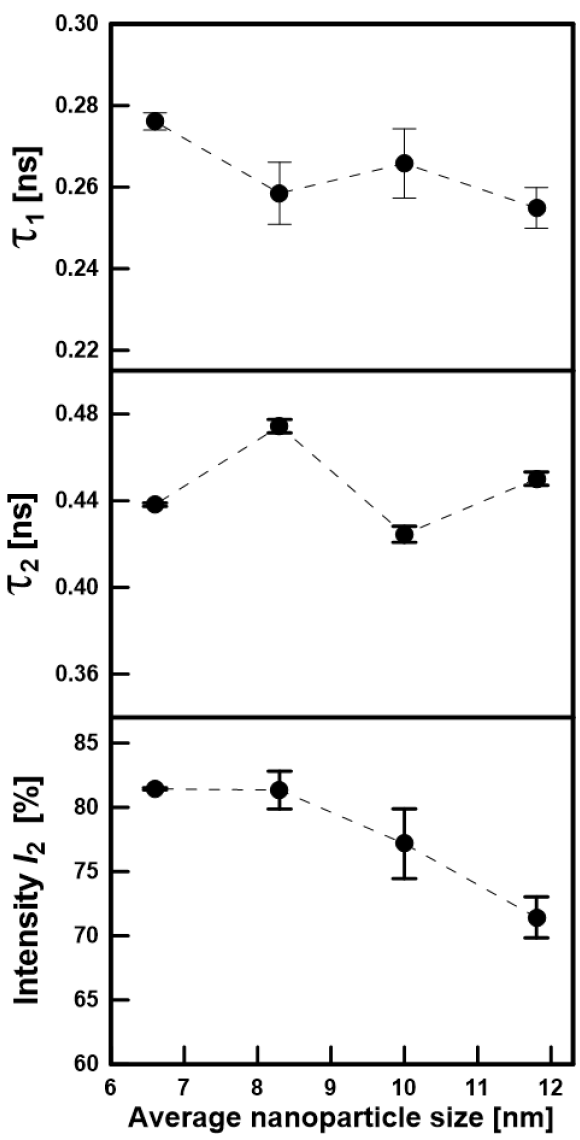

Fig. 4. The variations of the lifetimes $\tau_{1}, \tau_{2}$ and intensity of second component $I_{2}$ versus nanoparticle size for magnetite samples.

Values of the both lifetimes are practically constant. Only slight fluctuations in respect of their mean values (no more than $8 \%$ and $10 \%$, respectively) of the both lifetimes have been found. They may result from statistical uncertainties (in fitting procedure all positron annihilation parameters were not fixed). Alternatively, it is possible that not large drop observed for the $\tau_{1}$ component is caused by a small reducing of vacancy cluster volumes. The first component, $\tau_{1}$, is related to positrons trapped in free volumes in the region of grain boundaries (GB) [14]. In support of this interpretation the results of calculations carried out in [15] can also be cited. The second component corresponds to positrons trapped in nanovoids located in triple points [14]. Its intensity $I_{2}$ monotonically decreases from about $82 \%$ for nanoparticles of mean size $6.6 \mathrm{~nm}$ to $72 \%$ for that of $11.8 \mathrm{~nm}$. This tendency reflects the decrease the total volume connected with intergranular regions in comparison to the GB volume fraction with the increase of the average nanoparticle size.

In the PALS spectra of the nanopowder samples a lifetime corresponding to reduced bulk component has not been observed. Bulk lifetime $\tau_{b}$ measured in $\mathrm{Fe}_{3} \mathrm{O}_{4}$ natural monocrystals amounts to about 174 ps [16] or to 180(4) ps [17]. 
In the framework of the presented investigation the PALS measurement for polycrystalline magnetite powder (Alfa Aesar-325 mesh, average size of grains $44 \mu \mathrm{m}$ ) was also performed. Two components with positron lifetimes $\tau_{1}=142.5(8) \mathrm{ps}$ and $\tau_{2}=289.0(2.6) \mathrm{ps}$ had been found. Their relative intensities were equal to $I_{1}=66.72(87) \%$ and $I_{2}=32.80(70) \%$, respectively. Calculated on the basis of two-state trapping model [18] bulk lifetime $\tau_{b}=171(2)$ ps is in a good agreement with literature data [16, 17]. Moreover, in the both cases, in natural monocrystals and in the polycrystalline magnetite the second component with the lifetime in the range of $289 \div 297$ ps $[16,17]$ was registered. The nature of defects responsible for positron trapping and giving such lifetimes is not clear, whilst these defects occur in the bulk material and probably they are small clusters composed of a few vacancies.

The lifetimes measured for nanocrystalline magnetite (224(3) ps and 408(3) ps) were reported in [19]. They are slightly shorter than those obtained in our study but the mean size of nanocrystallites used in [19] was greater (about $31 \mathrm{~nm}$ ).

It is interesting that accordingly to available experimental data on positron annihilation in other spinel ferrite nanopowders of the same range of grain sizes the reduced bulk components have been observed [20-24]. It should be noted that in our measurements the samples containing free-flowing powders were used whilst in the cited papers compacted powder had been studied. The differences of similar character for results analogous measurements in free-flowing and compacted nanosized polycrystalline iron were reported in [14].

Absolute trapping rates and concentrations for observed two types defects could not be determined on the basis three-state trapping model [25] due to an occurrence of trapping saturation phenomenon. It is known that the diffused trapping model (DTM) is used to interpretation of PALS data for powder samples [26]. However, average grain size in studied magnetite samples is considerably lower than a typical diffusion length of positron in any bulk material $(100 \div 200 \mathrm{~nm})$. In such case the trapping rate of positrons at the grain boundary of $\mathrm{Fe}_{3} \mathrm{O}_{4}$ nanocrystallites becomes so high that only lifetimes longer than that of the free delocalized state are observed indicating saturation. Then the DTM is not applicable to more quantitative analysis of results and it suffices to state in this respect that annihilation takes place from states of positrons trapped in grain boundary defects.

In Fig. 5 the dependence of an average lifetime defined as:

$$
\tau_{\text {avg }}=\left(I_{1} \tau_{1}+I_{2} \tau_{2}\right) /\left(I_{1}+I_{2}\right)
$$

on mean nanoparticle size is shown. This parameter in a synthetic manner characterizes all annihilation processes occurring in the sample. It is independent of the number of lifetime components in deconvolution of PALS spectrum and therefore it is the most universal characteristics of annihilation.

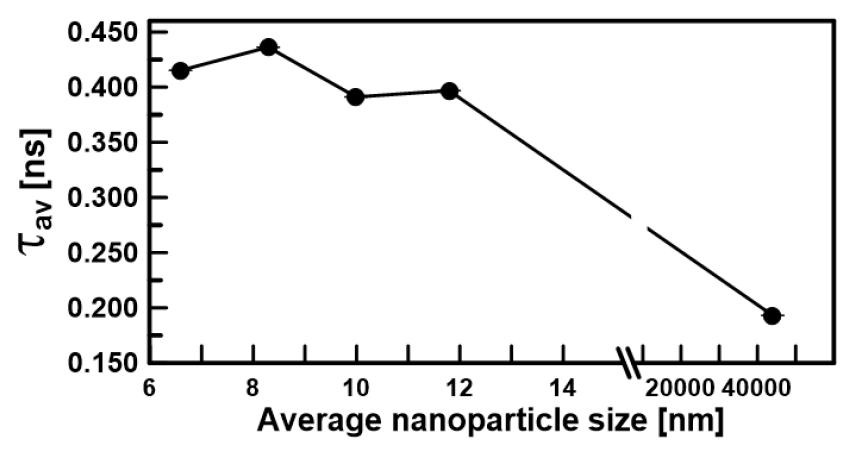

Fig. 5. The dependence of average positron annihilation lifetime $\tau_{a v g}$ on mean magnetite particle size.

As might be expected, $\tau_{a v g}$ monotonically decrease with an increase of the mean particle size in a very wide range from a few $\mathrm{nm}$ to tens of $\mu \mathrm{m}$.

Calorimetric measurements were performed for the sample S2. The raise of the sample temperature with an increase of the time of exposure of the sample to an $\mathrm{AC}$ magnetic field at an amplitude of $3.8 \mathrm{kA} / \mathrm{m}$, frequency of $177 \mathrm{kHz}$ was investigated. Data acquisition for hyperthermia was started at ambient temperature. The sample was prepared as described in the experimental section and dispersed in deionized water. The test tube of $2 \mathrm{ml}$ volume containing suspension of nanoparticles was placed in an insulating jacket made of polystyrene. Into the interior there was introduced the sensor fiber of thermometer in order to record changes of ferrofluid temperature as a function of time. Data acquisition for hyperthermia was started at ambient temperature. The sample was exposed to an alternating magnetic field for 5 min and then the field was turned off. As it was shown in Fig. 4, after 5 min the temperature optimal from the point of view of the hyperthermia effectiveness (about $42^{\circ} \mathrm{C}$ ) was reached. Turning off the magnetic field causes the temperature drop. The heating characteristic allows to determine specific absorption rate (SAR). The SAR is expressed as the heat released by the magnetic iron oxide nanoparticles subjected to the magnetic field. Its value was calculated from the formula [27]:

$$
S A R=C \frac{\Delta T}{\Delta t} \frac{\rho}{C_{n p}},
$$

where $C$ is the specific heat capacity of the medium $(4.18 \mathrm{~J} / \mathrm{K}), \Delta T / \Delta t$ is the initial slope of the timedependent temperature curve, $C_{n p}$ is the concentration of iron oxide nanoparticles in the medium $(60 \mathrm{mg} / \mathrm{mL})$ and $\rho$ is the density of the colloid suspension. The obtained values of the SAR for the sample $\mathrm{S} 2$ was equal to $21.5( \pm 2.5) \mathrm{W} / \mathrm{g}$.

Based on the characteristic shown in Fig. 6 and calculated value of SAR, it can be stated that the synthesized sample has a high capacity for heating up of a cancer tissue in an alternating magnetic field, showing the desired temperature increase in hyperthermia, just after a few minutes. 


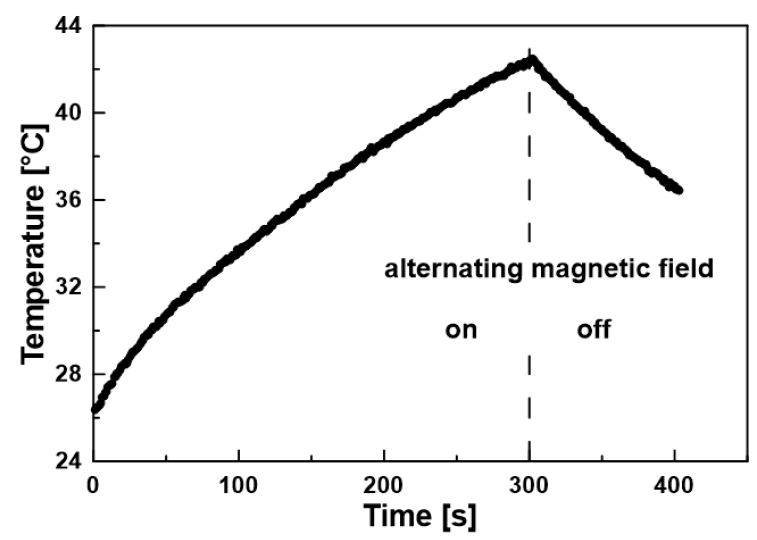

Fig. 6. Temperature rise as a function of time for the sample $2\left(H_{\max }=3.8 \mathrm{kA} / \mathrm{m}, f=177 \mathrm{kHz}\right)$.

\section{Conclusions}

Conclusions resulting from performed investigations can be summarized as follows:

1. Two main types of defects characterized by two main components found in PALS spectra: $\left(\tau_{1}, I_{1}\right)$ and $\left(\tau_{2}, I_{2}\right)$ exist in the nanosized $\mathrm{Fe}_{3} \mathrm{O}_{4}$ powders. They include large diffuse vacancy clusters or microvoids in disordered random grain-boundaries and intergranular spaces, so-called triple points.

2. Positron trapping saturation occurs in the investigated materials as a consequence of very high concentration of defects in them. The occurrence of saturation prevents the more detailed analysis of positron annihilation data and the quantitative determination of trapping rates and as consequence defects concentrations.

3. It seems that in spite of forming large aggregates of nanograins of magnetite in studied material the properties characteristic for nanopowders are preserved. Interactions between particular nanoparticles remains weak and in room temperature do not result in recrystallization processes.

4. Usefulness of the synthesized magnetite nanoparticles with dimensions about $8 \mathrm{~nm}$ for hyperthermia was approved.

\section{References}

[1] B. Chudzik, A. Miaskowski, Z. Surowiec, G. Czernel, T. Duluk, A. Marczuk, M. Gagoś, Int. J. Hypertherm. 32, 842 (2016).

[2] C. Billotey, C. Wilhelm, M. Devaud, C. Bacrij, J. Bittoun, F. Gazeau, Magn. Reson. Med. 49, 646 (2003).
[3] V.V. Mody, A. Cox, S. Shah, A. Singh, W. Bevins, H. Parihar, Appl. Nanosci. 4, 385 (2014).

[4] Q.A. Pankhurst, J. Connolly, S.K. Jones, J. Dobson, J. Phys. D Appl. Phys. 36, R167 (2003).

[5] M. Panczyk, Farmaceutyczny Przeglad Naukowy 10, 39 (2009) (in Polish).

[6] G.M. da Costa, E. de Grave, P.M.A. de Bakker, R.E. Vandenberghe, J. Solid State Chem. 113, 405 (1994).

[7] M. Tajabadi, M.E. Khosroshahi, MAPCBEE Proc. 3, 140 (2012).

[8] E.A. Perigo, G. Hemery, O. Sandre, D. Ortega, E. Garaio, F. Plazaola, F.J. Teran, J. Appl. Phys. 2, 041302 (2015).

[9] A. Hervault, Nguyen Thi Kim Thanh, Nanoscale 6, 11553 (2014).

[10] C.S.S.R. Kumar, F. Mohammad, Adv. Drug. Deliv. Rev. 63, 789 (2011).

[11] J.V. Olsen, P. Kirkegaard, N.J. Pedersen, M. Eldrup, Phys. Status Solidi C 4, 4004 (2007).

[12] W.P. Neves, C.R. Soares Sousa, A.L. Miranda-Vilela, G.L. Silva Santos, P.E.N. de Souza, J.P. Figueiró Longo, Z.G. Marques Lacava, J. Cancer Sci. Ther. 9, 393 (2017)

[13] F. Tuomisto, I. Makkonen, Rev. Mod. Phys. 85, 1583 (2013).

[14] H.E. Schaefer, R. Würschum, R. Birringer, H. Gleiter, Phys. Rev. B 38, 9545 (1988).

[15] J. Kuriplach, Acta Phys. Pol. A 125, 722 (2014).

[16] D. Das, M.B. Chakraborti, K. Choudhury, P.M.G. Nambissan, B.R.S. Babu, P. Sen, Sangeeta, C.K. Majumdar, Bull. Mater. Sci 15, 161 (1992).

[17] K. Uhlmann, D.T. Britton, S. Heger, Mater. Sci. Forum 175-178, 225 (1995).

[18] A. Seeger, Appl. Phys. 4, 183 (1974).

[19] A. Cabral-Prieto, A.A. Reyes-Felipe, M.G. SilesDotor, Nanostruct. Mater. 10, 311 (1998).

[20] P.M.G. Nambissan, C. Upadhyay, H.C. Verma, J. Appl. Phys. 93, 6320 (2003).

[21] S. Bandyopadhyay, A. Roy, D. Das, S.S. Ghugre, J. Ghose, Philos. Mag. 83, 765 (2003).

[22] S. Chakraverty, Subarna Mitra, K. Mandal, P.M.G. Nambissan, S. Chattopadhyay, Phys. Rev. B 71, 024115 (2005).

[23] P. Predeep, A.S. Prasad, S.N. Dolia, M.S. Dhawan, M.S. Das, S.K. Chaudhuri, Vipasha Ghose, IEEE Trans. Magn. 46, 847 (2010).

[24] H. Klym, A. Ingram, O. Shpotyuk, J. Filipecki, Visnyk Lviv Univ. Ser. Phys. N 40, 200 (2007).

[25] J. Kansy, D Giebel, J. Phys. Conf. Ser. 265, 012030 (2011).

[26] J. Dryzek, J. Phys. Condens. Matter 12, 137 (2000).

[27] G. Vallejo-Fernandez, O. Whear, A.G. Roca, S. Hussain, J. Timmis, V. Patel, K. O'Grady, J. Phys. D Appl. Phys. 46, 312001 (2013). 